\title{
AVALIAÇÃO DOS POTENCIAIS EVOCADOS RELACIONADOS A EVENTOS (ERP-P300) EM PACIENTES COM CIRROSE HEPÁTICA SEM ENCEFALOPATIA
}

\author{
Vinicius TEODORO',2, Maurício BRAGAGNOLO Jr. ${ }^{3}$, Lígia LUCCHESI², \\ Mário KONDO ${ }^{3}$ e Sérgio TUFIK ${ }^{2}$
}

\begin{abstract}
RESUMO - Racional - Na cirrose hepática ocorrem alterações na estrutura do fígado, levando à perda das funções do órgão, com conseqüências neuropsiquiátricas, como disfunções cognitivas. Um dos meios mais efetivos para avaliar objetivamente as funções cognitivas é medir a atividade eletrofisiológica do sistema nervoso central através do potencial evocado relacionado a eventos (ERP-P300). Objetivo - Estudar a utilidade do potencial evocado relacionado a eventos (ERP), para avaliar distúrbios cognitivos em pacientes com cirrose hepática e como auxiliar no diagnóstico da encefalopatia hepática mínima. Método - Foram selecionados 50 pacientes, diagnosticados com cirrose hepática sem sinais clínicos de encefalopatia hepática e 35 voluntários saudáveis de idades e sexo semelhantes. Em todos os pacientes foram realizados exames clínico-neurológico e laboratoriais. Para identificação do prejuízo cognitivo foi utilizado o ERP-P300 e obtida a média da latência da onda P300. Resultados - Houve diferença significativa entre as médias da latência do ERP-P300 do grupo cirrótico e controle. Conclusão - A realização do ERP-P300 é simples, depende de fatores controláveis de variação e tem fácil reprodutibilidade, podendo ser útil para o rastreio de distúrbios cognitivos em pacientes com cirrose e auxiliar no diagnóstico de encefalopatia hepática mínima.
\end{abstract}

DESCRITORES - Cirrose hepática. Encefalopatia hepática. Potenciais evocados.

\section{INTRODUÇÃO}

A cirrose hepática $(\mathrm{CH})$ é um processo crônico e irreversível, resultado do aumento difuso da atividade fibroblástica no fígado, em resposta a determinadas agressões continuas ao órgão ${ }^{(19)}$. A fibrose resulta da cicatrização que se segue à destruição do hepatócito e com o colapso da trama de reticulina que o sustenta, resultando na desorganização da arquitetura hepática. A alteração na estrutura hepática faz compressão dos ramos terminais da veia porta, provocando aumento da resistência local. O aumento da pressão local provoca o desvio do fluxo sangüíneo venoso proveniente das veias mesentérica superior e esplênica, rico em substâncias resultantes da digestão, que deixam de ser metabolizadas pelo fígado e acabam circulando diretamente para o sistema nervoso central $^{(32)}$. Os pacientes cirróticos podem apresentar um ou dois tipos de manifestações clinicas: as relacionadas diretamente à necrose hepatocelular, causando perda da função hepática e sinais ou sintomas das complicações da cirrose devido à hipertensão portal. A evolução do paciente é assintomática e insidiosa, marcada por sintomas inespecíficos (anorexia, perda de peso, fraqueza, osteoporose), dificultando o diagnóstico precoce. As principais causas da cirrose hepática são os agentes tóxico-químicos (álcool e drogas) e as infecções virais crônicas (hepatites B e C) ${ }^{(1)}$. Atualmente há aumento do número de pacientes com cirrose decorrente de hepatite C, estimando-se em 200 milhões de portadores do vírus no mundo ${ }^{(31)}$. O diagnóstico de cirrose e sua incidência têm crescido em nosso meio, tendo sido responsável por 40.000 internações hospitalares e mortalidade de 7,79 por 100 mil habitantes em $2002^{(4)}$.

As alterações neuropsiquiátricas, agudas ou crônicas, decorrentes da disfunção hepática e/ou da hipertensão portal, podem ser sumarizadas como encefalopatia hepática $(\mathrm{EH})^{(6)}$. As alterações cognitivas, comportamentais e motoras mais comumente encontradas na EH manifesta, incluem desde distúrbios mínimos de atenção, de memória, de sono ${ }^{(12)}$ e motores (distúrbios extrapiramidais), até quadros mais graves de comprometimento do sistema nervoso central com torpor, delírio, confusão mental e

Apoio financeiro: AFIP, FAPESP (CEPID)

'Departamento de Gastroenterologia; 'Departamento de Psicobiologia da Universidade Federal de São Paulo - Escola Paulista de Medicina, São Paulo, SP.

Correspondência: Dr. Vinicius Teodoro - Rua João Castaldi, 65 - apt. 91 - Indianópolis - 04517-050 - São Paulo, SP. Email: viniteodoro@uol.com.br 
coma $^{(2,8)}$. O quadro inicial de EH, no qual não são encontradas alterações ao exame clínico-neurológico, é denominado de encefalopatia hepática mínima (EHM) ou latente ${ }^{(21)}$. As atividades motoras e intelectuais mais complexas, como conduzir veículos ou aprender novas tarefas são afetadas desde estágios iniciais da cirrose $^{(37)}$, já indicando algum prejuízo cognitivo. As dificuldades no reconhecimento destas alterações, inicialmente sutis, levaram à elaboração de diversas baterias de testes ${ }^{(18,30,33)}$ para seu correto diagnóstico. FERENCI e LOCKWOOD ${ }^{(10)}$ em uma força tarefa, sugeriram o uso de testes neuropsicológicos (teste de conexão numérica A e B, Digit Symbol ou Digit Span) e, quando possível, associar testes neurofisiológicos (ERP-P300 e EEG) para o diagnóstico da EHM.

Um dos meios mais efetivos para avaliar objetivamente as funções cognitivas é medir a atividade eletrofisiológica do sistema nervoso central através dos $\operatorname{ERP}^{(20)}$, que constitui instrumento valioso para estudar a atividade neuronal gerada durante o processamento de novas informações ${ }^{(26)}$. O teste mais freqüentemente usado para provocar o aparecimento do ERP é a tarefa auditiva discriminatória utilizando o paradigma "oddball", na qual é solicitado aos testados detectar estímulos auditivos alvo e ocasionais, intercalados entre estímulos auditivos padrões mais freqüentes ${ }^{(5)}$. Mudanças na latência do componente P3 ou P300 têm sido associadas com variáveis cognitivas como memória ${ }^{(9)}$, tomada de decisão ${ }^{(15)}$ e expectativa(7).

Diversos estudos utilizaram o ERP-P300 para a avaliação dos distúrbios cognitivos em pacientes com cirrose. WEISSENBORN et al. ${ }^{(38)}$ e DAVIES et al. ${ }^{(8)}$, em 1990, publicaram os primeiros trabalhos utilizando o ERP-P300 com esta finalidade. Entretanto, estudos posteriores $^{(18,33,34)}$ que também utilizaram o ERP-P300 com o mesmo objetivo, obtiveram resultados diferentes, com número elevado de cirróticos com EHM. De acordo com SAXENA et al. ${ }^{(33)}$, há uma variabilidade importante da incidência de EH e da EHM, atingindo até $84 \%$ dos pacientes com cirrose, dependendo do método utilizado para seu diagnóstico.

A presença de distúrbios cognitivos na encefalopatia hepática tem efeito negativo sobre a qualidade de vida e o prognóstico dos pacientes com cirrose ${ }^{(24,30)}$. Entretanto, não se dispõe ainda de métodos diagnósticos ditos padrão-ouro para diagnosticar essa síndrome, principalmente em seus estágios iniciais ${ }^{(16)}$.

O objetivo deste estudo foi verificar a utilidade do uso do ERP-P300, para avaliar os distúrbios cognitivos presentes nos pacientes com cirrose hepática sem encefalopatia e comparalos com um grupo controle. A utilização deste instrumento em pacientes com encefalopatia hepática foi amplamente descrita na literatura ${ }^{(18,33,34)}$ e é eminentemente clínica, razão pela qual optou-se por estudar somente pacientes sem sinais de alterações ao exame clínico-neurológico.

\section{MÉTODOS}

O protocolo de pesquisa foi avaliado e aprovado pelo Comitê de Ética da Universidade Federal de São Paulo - Escola Paulista de Medicina, São Paulo, SP. Todos os pacientes assinaram o Termo de Consentimento Livre e Esclarecido, concordando em participar da pesquisa.
No período de maio 2003 a maio de 2005 foram selecionados 50 pacientes (40 homens), de idade entre 31 e 65 anos (Tabela 1), diagnosticados com cirrose hepática e sem sinais clínicos de encefalopatia hepática. Todos foram provenientes do ambulatório de cirrose hepática, do Serviço de Gastroenterologia do Hospital São Paulo, São Paulo, SP. O diagnóstico de cirrose hepática foi feito através de exames clínicos, laboratoriais, ultra-sonografia ou exame histopatológico, quando possível. Os critérios de exclusão utilizados foram os seguintes: sangramento digestivo nos últimos 6 meses, insuficiência renal (definida como creatinina sérica $>2,0 \mathrm{mg} / \mathrm{dL}$ ), diagnóstico de carcinoma hepatocelular, uso de benzodiazepínicos nas últimas 2 semanas, uso de álcool nos últimos 6 meses, idade inferior a 18 anos ou superior a 65 anos. $\mathrm{O}$ grupo controle foi formado por 35 voluntários sadios com idade entre 20 e 65 anos selecionados entre os familiares saudáveis dos pacientes e obedecendo aos mesmos critérios de exclusão pré-estabelecidos para o grupo cirrótico.

TABELA 1. Resultados laboratoriais do grupo com cirrose hepática

\begin{tabular}{lcccc}
\hline \multirow{2}{*}{ Exames laboratoriais } & \multicolumn{4}{c}{ Resultados } \\
\cline { 2 - 5 } & Média & Desvio padrão & Mínimo & Máximo \\
\hline Sódio (mmol/L) & 140,5 & 3,1 & 133,0 & 151,0 \\
Potássio (mmol/L) & 4,5 & 0,1 & 3,6 & 5,8 \\
Bilirrubinas totais (mg/dL) & 2,2 & 2,9 & 0,5 & 21,1 \\
Bilirrubina direta (mg/dL) & 0,8 & 0,5 & 0,1 & 2,1 \\
Creatinina (mg/dL) & 0,9 & 0,3 & 0,5 & 1,8 \\
TGO (U/L) & 66,1 & 38,9 & 23 & 183 \\
TGP (U/L) & 62,8 & 60,5 & 16 & 417 \\
Fosfatase alcalina (U/L) & 142,0 & 69,1 & 43 & 308 \\
Gama GT (U/L) & 97,2 & 66,8 & 22 & 368 \\
Albumina (mg/dL) & 3,4 & 0,6 & 0,6 & 4,9 \\
Atividade protrombínica (\%) & 72,2 & 18,5 & 19,4 & 100 \\
Amônia arterial & 175,3 & 152,6 & 33,0 & 542 \\
(valores de referência: 9-33 mmol/L) & & & & \\
\hline
\end{tabular}

A avaliação inicial incluiu anamnese detalhada, avaliação da classe socioeconômica através do critério ABA/ABIPEME (Associação Brasileira de Anunciantes/Associação Brasileira de Institutos de Pesquisa de Mercado), exame clínico geral, exame neurológico e psiquiátrico convencional, exames laboratoriais e definição da etiologia da cirrose, quando possível. Os exames laboratoriais realizados após jejum de 12 horas e também seus resultados constam da Tabela 2. A classificação de Child-Pugh ${ }^{(29)}$ foi utilizada como forma de diferenciação da gravidade entre os grupos (Tabela 1).

O ERP-P300 foi realizado sempre pelo mesmo examinador, num mesmo ambiente, com iluminação e temperatura padronizadas, sempre no mesmo horário ( 9 horas da manhã), para evitar variação circadiana $^{(25)}$. Eletrodos ativos foram colocados nas posições $\mathrm{FZ}, \mathrm{CZ}$ e PZ, de acordo com o sistema internacional 10-20. Eletrodos de referência foram colocados em A1 e A2, ligados entre si. O eletrodo terra foi posicionado em FPZ. Foram também colocados eletrodos 
acima da sobrancelha direita e no canto externo do olho esquerdo para controle do eletro-oculograma. O paradigma utilizado foi o "oddball". Os estímulos auditivos foram apresentados bilateralmente através de fones de ouvido com tons de $70 \mathrm{~dB}$ e dois tons foram apresentados de forma aleatória: estímulo raro, de $1500 \mathrm{~Hz}$ (com $20 \%$ de probabilidade de aparecimento) e estímulo freqüente, de $800 \mathrm{~Hz}$ (com $80 \%$ de probabilidade de aparecimento). O intervalo entre os estímulos foi fixado em 2 segundos. Foi pedido ao testado que apertasse um botão todas as vezes que escutasse um som raro, com a mão dominante, tão rapidamente quanto possível. Foram obtidas médias de 30 registros de estímulos raros, livres de artefatos. Todos os procedimentos foram repetidos, para permitir a replicação das ondas obtidas e facilitar a identificação dos componentes.

TABELA 2. Características do grupo estudado

\begin{tabular}{lcc}
\hline Sexo & Feminino & Masculino \\
\hline Grupo doente $(\mathrm{n}=50)$ & 10 & 40 \\
Grupo controle $(\mathrm{n}=35)$ & 10 & 25 \\
\hline Etiologia da cirrose $(\mathrm{n}=50)$ & Número de pacientes \\
Alcoólica & 22 \\
Alcoólica associada à viral & 10 \\
Viral & 14 \\
Criptogênica & 4 \\
\hline Classificação de Child $(\mathrm{n}=50)$ & Número de pacientes \\
A & 21 \\
B & 19 \\
C & 10 \\
\hline
\end{tabular}

Para comparação das médias das latências, optou-se por dividir os grupos conforme faixa etária, devido à conhecida influência da idade sobre a latência do P300 ${ }^{(11,25)}$. Dividiu-se o grupo doente (D), em D1 ( $n=15)$ com idade de 25 a 45 anos, e D2 $(n=35)$ com idade entre 46 a 65 anos. Da mesma forma, foi dividido o grupo controle (C), em C1 ( $=19)$ e C2 $(n=16)$. Para a construção do banco de dados e cálculos estatísticos, foi utilizado o programa Statistica da Stat Soft 1995-2001. As variáveis foram expressas como média \pm desvio padrão e todos os grupos estudados apresentavam distribuição com curva normal pelo teste de Kolmogorov e Sminorv. Para a comparação das variáveis entre o grupo estudado e o grupo controle foi utilizado o teste $t$ de Student para amostras independentes ou análise de variância. Para o estudo de correlações entre as variáveis foi utilizado o teste de correlação de Spearman. O nível de significância de 0,05 foi utilizado em todos os testes.

\section{RESULTADOS}

Os pacientes com $\mathrm{CH}$ no presente estudo obtiveram a média da latência do ERP-P300 estatisticamente superior às médias obtidas no grupo controle (Figura 1). A latência média do grupo D1 foi de 349,69 ms versus grupo $\mathrm{C} 1$ com $328,10 \mathrm{~ms}$ e com valores de $t=2,29 \operatorname{com} P=0,03$. A latência do grupo mais idoso (D2) foi de 372,41 ms versus C2 de 344,62 ms e de $t=2,56 \operatorname{com} P=0,01$ (Tabela 3).

Para verificar se o uso crônico de álcool em uma parte da presente amostra de pacientes com cirrose teria influência nos resultados do ERP-P300, o grupo de cirróticos foi subdividido

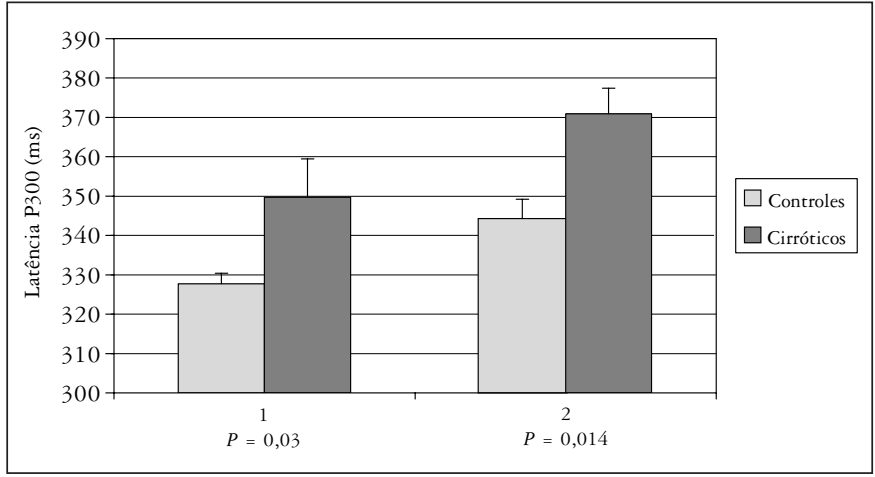

FIGURA 1. Média \pm erro padrão dos grupos controle e cirrose, divididos por idade: $25-45$ anos (1) e de 46-65 anos (2)

TABELA 3. Resultados

\begin{tabular}{cc}
\hline Grupos (divididos por idade) & Média e desvio padrão (anos) \\
\hline Grupo D $(\mathrm{n}=50)$ & $49,6 \pm 8,3$ \\
Grupo D1 $(\mathrm{n}=15)$ & $36,0 \pm 6,8$ \\
Grupo D2 $(\mathrm{n}=35)$ & $53,9 \pm 5,3$ \\
Grupo C $(\mathrm{n}=35)$ & $46,6 \pm 9,4$ \\
Grupo C1 $(\mathrm{n}=19)$ & $39,2 \pm 4,0$ \\
Grupo C2 $(\mathrm{n}=16)$ & $54,1 \pm 4,8$ \\
\hline Latência do ERP-P300 & Média e desvio padrão(ms) \\
Grupo D1 $(\mathrm{n}=15)$ & $346,40 \pm 37,09$ \\
Grupo D2 $(\mathrm{n}=35)$ & $374,26 \pm 41,17$ \\
Grupo C1 $(\mathrm{n}=19)$ & $344,10 \pm 11,31$ \\
Grupo C2 $(\mathrm{n}=16)$ & $\pm 21,75$ \\
\hline Grupo de pacientes cirróticos $(\mathrm{D})$ dividido em D1 com idade entre 25 e 45 anos e D2 entre 46 e 65 anos \\
Grupo controle $(\mathrm{C}$ ) dividido em Cl com idade entre 26 e 45 anos e C2 entre 46 e 65 anos
\end{tabular}

Grupo controle(C) dividido em $\mathrm{Cl}$ com idade entre 26 e 45 anos e $\mathrm{C} 2$ entre 46 e 65 anos

em dois subgrupos: o primeiro foi formado por pacientes com história prévia de uso abusivo de álcool e o segundo, por pacientes sem história prévia de uso abusivo de álcool. Após a divisão utilizou-se o teste $t$ de Student para a comparação das médias das latências do ERP-P300 entre os dois subgrupos e não foi observada diferença estatisticamente significante entre eles $(P=0,67)$. Da mesma forma, a gravidade da disfunção hepática, avaliada pela classificação Child-Pugh não interferiu significantemente nos valores médios da latência da onda ERPP300 no grupo avaliado $(P=0,38)$. Também não foi encontrada correlação significativa entre amônia e a latência do ERP-P300 no grupo de pacientes com cirrose $(r=0,26)$.

\section{DISCUSSÃO}

Desde a primeira descrição da onda P300 por SUTTON et al. ${ }^{(35)}$, em 1965, muito se tem aprendido sobre o significado deste componente. A utilização do ERP-P300 como método de avaliação e rastreio dos prejuízos na função cognitiva, vem a cada dia aumentando seu campo de aplicação. A geração da onda P300 do ERP é complexa e, provavelmente, envolve diferentes centros e circuitos neuronais. Inicialmente foi sugerido por HALGREN et al. ${ }^{(13)}$ e McCARTHY et al. ${ }^{(22)}$ que a geração da onda P300 seria em áreas hipocampais do lobo têmporo-medial, entretanto estudos subseqüentes demonstraram que a formação hipocampal apenas influenciaria indiretamente a geração do 
potencial P300 ${ }^{(23)}$. Atualmente a junção têmporo-parietal ${ }^{(17)}$ vem sendo reconhecida como região principal para a geração desse potencial. Resumidamente, o estimulo neural inicia-se em áreas fronto-córtico-subcorticais ${ }^{(28)} \mathrm{e}$, possivelmente, se irradia para o outro hemisfério via corpo caloso ${ }^{(28)}$, chegando às áreas associativas hipocampais subcorticais centrais e parietais do lobo temporal, representando o período de resposta do córtex de associação ao estimulo proveniente do córtex primário ${ }^{(36)}$. O gerador ou geradores específicos ainda permanecem incertos.

WEISSENBORN et al. ${ }^{(38)}$ utilizaram diversos testes neurofisiológicos (EEG, ERP-P300 e potencial visual evocado) em 66 pacientes com cirrose hepática de diversas causas, excluindo as provocadas por álcool ou tóxicas e encontraram prolongamento da latência do ERP-P300 em 90\% dos pacientes nos estágios iniciais de $\mathrm{EH}$, sendo 19\% dos pacientes sem sinais clínicos de EH. Esse estudo demonstrou a sensibilidade do ERP-P300 para o diagnóstico da $\mathrm{EH}$, principalmente em seus estágios iniciais. $\mathrm{O}$ trabalho de DAVIES et al. ${ }^{(8)}$, que utilizaram potenciais ERPP300, EEG e teste de conexão numérica-A em 33 cirróticos de diversas causas, também descreveram latência aumentada do ERP-P300 no grupo com cirrose e com sinais clínicos de EH. Nesse trabalho não foi encontrada diferença significante entre o grupo com cirrose sem sinais clínicos de EH e o grupo controle, diferentemente do que foi encontrado por WEISSENBORN et al. ${ }^{(38)}$. Em estudo recente, HOLLERBACH et al. ${ }^{(14)}$ investigaram 45 pacientes com cirrose, mas sem história prévia de uso abusivo de álcool, e encontraram atraso na latência da onda P300 em 35\% deles, dos quais 44\% não apresentaram sinais clínicos de EH.

Os diversos estudos apresentados valorizam o resultado do ERP-P300, entretanto a variação nos critérios de inclusão e exclusão utilizados, bem como do modelo do experimento, levou ao número alto na variação das percentagens de pacientes cirróticos sem sintomas clínicos de $\mathrm{EH}$, mas com alteração no ERP-P300, como descrito por SAXENA et al. ${ }^{(34)}$ que, ao avaliarem diversos estudos em cirróticos, observaram variação de $30 \%$ até $84 \%$ na prevalência de EHM nesse grupo. Conclui-se que essa variação dependeria principalmente do método utilizado para o diagnóstico da $\mathrm{EH}$, dos diferentes critérios de inclusão e exclusão e independeria da causa da cirrose $^{(34)}$. A utilização de testes padronizados ajudaria a diminuir essa variação. No grupo estudado, a causa da cirrose não influenciou os resultados do ERP-P300, como demonstrado em outros estudos ${ }^{(8,27)}$. Também devem ser considerados fatores de variabilidade como temperatura, nível de luz e ritmo circadiano ${ }^{(25)}$.

Sumarizando, a utilização de diversas baterias de testes neurofisiológicos e neuropsicológicos para o diagnóstico dos distúrbios cognitivos nos pacientes com cirrose, resulta em importante variação na sua incidência. $\mathrm{O}$ uso apenas de testes neuropsicológicos, como preconizados por WEISSENBORN et al. ${ }^{(38)}$, usando apenas lápis e papel, é interessante, mas são testes que necessitam de devida normatização, muitos deles influenciados pela baixa escolaridade ${ }^{(3)}$ e dependem de correção para a idade. A possibilidade de se utilizar um teste simples e independente de fatores de variação importantes, facilitaria o diagnóstico da EHM e diminuiria a variação encontrada na sua incidência. O ERP-P300 devidamente utilizado fornece informações importantes sobre a função cognitiva do paciente e a sua latência é uma medida do tempo de processamento de informações que, no grupo cirrótico, apresentou retardo significativo quando comparado ao grupo controle. Este retardo poderia estar ligado a um déficit de memória de curto prazo, atenção e concentração ${ }^{(9,25)}$. É, também, um instrumento de utilização simples e não influenciado diretamente pelo nível de escolaridade ${ }^{(36)}$. De acordo com diversos estudos bem conduzidos, a origem da cirrose (alcoólica versus outras) parece não interferir na comparação das médias da latência do ERP-P300 entre os grupos ${ }^{(8,27)}$.

\section{CONCLUSÃO}

Os achados do presente estudo demonstram a utilidade do ERP-P300 para o rastreio e diagnóstico de EHM nos pacientes com cirrose hepática. Além disso, a realização do ERP-P300 é simples, depende de fatores controláveis de variação e tem fácil reprodutibilidade.

Teodoro V, Bragagnolo Jr M, Lucchesi L, Kondo M, Tufik S. Evaluation of the event-related potential (ERP-P300) in patients with hepatic cirrhosis without encephalopathy. Arq Gastroenterol. 2008;45(1):82-6.

ABSTRACT - Background - In hepatic cirrhosis structural liver alterations occur leading to the loss of the organ functions with neuro-psychiatric consequence, as cognitive dysfunctions. One of the most effective ways of objectively evaluating cognition is to measure electrophysiological activity in the central nervous system trough event-related potentials (ERP-P300). Aim - To assess the value of the event-related potential (ERP) in order to determine cognitive disturbances in patients with liver cirrhosis and to assist in the diagnosis of minimal hepatic encephalopathy. Methods - Fifty patients with liver cirrhosis were selected, without clinical symptoms of hepatic encephalopathy and 35 healthy volunteers, matched by sex and age. The patients were submitted to clinical-neurological and laboratorial examination. The ERP-P300 was performed by the two groups to determine cognitive disturbances. Results - The study showed significant differences between the ERP-P300 latency averages of the two groups. Conclusion - The ERP-P300 is simple to use and depends on controllable variables. It is also easy to reproduce and, when properly used, can be useful both to determine cognitive disturbances in patients with hepatic cirrhosis and to assist in minimal hepatic encephalopathy diagnosis.

HEADINGS - Liver cirrhosis. Hepatic encephalopathy. Evoked potentials. 


\section{REFERÊNCIAS}

1. Alter MJ, Kruszon-Moran D, Nainan OV, McQuillan GM, Gao F, Moyer LA, Kaslow RA, Margolis HS. The prevalence of hepatitis C virus infection in the United States, 1988 through 1994. N Engl J Med. 1999;341:556-62.

2. Blei A, Cordoba J. Hepatic encephalopathy. Am J Gastroenterol. 2001;96:1968-75.

3. Bragagnolo Jr MA. Diagnóstico de encefalopatia hepática mínima através de testes neuropsicológicos e neurofisiológicos [tese]. São Paulo: Universidade Federal de São Paulo - Escola Paulista de Medicina; 2005.

4. Brasil. Ministério da Saúde. DATASUS.[online] Informações de saúde. (Disponível em URL: http://www.datasus.gov.br.)

5. Celesia GG, BrigelI M. Event-related potentials. Curr Opin Neurol Neurosurg. 1992;5:733-9.

6. Conn HO, Levy ZB, Vlahcevic JB, Rodgers WC, Madray C, Seeff C, Levy LL. Comparison of lactulose and neomycin in the treatment of chronic portal systemic encephalopathy. Gastroenterology. 1977;72:573-83.

7. Coull JT. Neural correlates of attention and arousal: insights from electrophysiology, functional neuroimaging and psychopharmacology. Prog Neurobiol. 1998;55:343-61.

8. Davies MG, Rowan MJ, MacMathuna P, Napoleon KP, Weir D, Feely J. The auditory P300 event-related potential: an objective marker of the encephalopathy of chronic liver disease. Hepatology. 1990;12:688-94.

9. Donchin E. Surprise! ...surprise? Psychophysiology. 1981;18:493-513.

10. Ferenci P, Lockwood A, Mullen K, Tarter B, Weissenborn K, Blei AT. Hepatic encephalopathy - definition, nomenclature, diagnosis and quantification: final report of the working party at the $11^{\text {th }}$ World Congresses of Gastroenterology, Vienna, 1998. Hepatology. 2002;35:716-21

11. Goodin DS, Squires KC, Henderson BH, Starr A. Age related variations in evoked potentials to auditory stimuli in normal human subjects. Electoencephalogr Clin Neurophysiol. 1978;44:447-58.

12. Groeneweg M, Quero JC, Bruijin IJC, Essink-Bot ML, Hop WCJ. Subclinical hepatic encephalopathy impairs daily functioning. Hepatology. 1998;28:45-9.

13. Halgren E, Squires N, Wilson C, Rohrbaugh J ,Bab T, Crandall P. Endogenous potentials in the human hippocampal formation and amygdala by infrequent events. Science. 1980;210:803-5.

14. Hollerbach S. Kullmann F, Fründ R, Lock G, Geissler A, Scholmerich J, Holstege A. Auditory event-related cerebral potentials (P300) in hepatic encephalopathy: topographic distribution and correlation with clinical and psychometric assessment. Hepatogatroenterology. 1997;44:1002-12.

15. Iragui VJ, Kutas M, Mitchiner MR, Hillyard AS. Effects of aging on event-related brain potentials and reaction times in an auditory oddball task. Psychophysiology. 1993;30:10-22.

16. Kharbanda P, Prabhakar S. Subclinical hepatic encephalopathy: elusive gold standard. J Gastroenterol Hepatol. 2004;19:1-3.

17. Knight RT, Scabini D, Woods DL, Clayworth CC. Contributions of temporal-parietal junction to the human auditory P3. Brain Res. 1989;502:109-16.

18. Kulmann F, Hollerbach S, Holstege, Schomerich J. Subclinical hepatic encephalopathy: the diagnostic value of evoked potentials. J Hepatol. 1995;22:101-10.

19. Lida VH, Silva TJ, Silva ASF, Silva LFF, Alves VAF. Cirrose hepática: aspectos morfológicos relacionados às suas possíveis complicações. Um estudo centrado em necropsias. J Bras Patol Med Lab. 2005;41:29-36.
20. Lucchesi LM, Braga NI, Manzano GM, Pompeia S, Tufik S. Acute neurophysiological effects of the hypnotic zolpidem in healthy volunteers. Prog Neuropsychopharmacol Biol Psychiatry. 2005;29:557-64.

21. Mattarozzi K, Stracciari A, Vignatelli L, Alessandro R, Morelli MC, Guarino M. Minimal hepatic encephalopathy. Arch Neurol. 2004;61:242-7.

22. McCarthy G, Wood CC, Williamson PD, Spencer DD. Task-dependent field potentials in human hippocampal formation. J Neurosci. 1989;9:4253-68.

23. Molnar M. On the origin of the P300 event-related potential component. Int $\mathrm{J}$ Psychophysiol. 1994;17:129-44.

24. Pereira HMV, Cavalheiro N, Tengan FM, Melo CE, Mello ES, Barone AA. Pacientes com hepatite $\mathrm{C}$ crônica e transaminases normais. Rev Inst Med Trop S Paulo. 2005;47:247-50.

25. Polich J. P-300 clinical utility and control of variability. J Clin Neurophysiol. 1998;1:14-33.

26. Pooviboonsuk P, Dalton JA, Curran HV, Lader MH. The effects of single doses of lorazepam on event-related potentials and cognitive function. Hum Psychopharmacol. 1996;11:241-52.

27. Porjesz B, Rangaswamy M, Kamarajan C, Jones KA, Padmanabhapillai A, Begleiter H. The utility of neuropsychological markers in the study of alcoholism. Clin Neurophysiol. 2005;116:993-1018.

28. Posner MI, Petersen SE. The attention system of the human brain. Annu Rev Neurosci. 1990; $13: 25-42$.

29. Pugh RN, Murray-Lyon IM, Nunnerley HB, Laws JW, Dawson JL, Williams R. Treatment of bleeding oesophageal varices by infusion of vasopressin into the superior mesenteric artery. Gut. 1973;14:59-63.

30. Quero JC, Schalm SW. Subclinical hepatic encephalopathy. Semin Liver Dis 1996; $16: 321-8$

31. Riestra S, Fernandez E, Leiva P, Garcia S, Ocio G, Rodrigo L. Prevalence of hepatitis $\mathrm{C}$ virus infection in the general population of northern Spain. J Gastroenterol Hepatol. 2001;13:477-81.

32. Rigas B, Spiro HM. Distúrbios hepáticos. In: Rigas B, Spiro HM. Gastroenterologia clinica $4^{\mathrm{a}}$ ed. Rio de Janeiro: McGraw-Hill; 1996. p.517-618.

33. Saxena N, Bathia M, Joshi YK, Garg PK, Tandon RK. Auditory P300 event-related potentials and number connection test for evaluation of subclinical hepatic encephalopathy in patients with cirrhosis of the liver: a follow-up study. J Gastroenterol Hepatol. 2001;16:322-7.

34. Saxena N, Bathia M, Joshi YK, Garg PK, Dwivedi SN, Tandon RK. Electrophysiologica and neuropsychological tests for the diagnosis of subclinical hepatic encephalopathy and prediction of overt encephalopathy. Liver. 2002;22:190-7.

35. Sutton NS, Braren M, John ER. Evoked potentials correlates of stimulus uncertainty Science. 1965;150:1187-8.

36. Visioli-Melo JF, Rotta NT. Avaliação pelo P300 de crianças com e sem epilepsia e rendimento escolar. Arq Neuropsiq. 2000;58:476-84.

37. Wein C, Koch H, Popp B, Oehler G, Schauder P. Minimal hepatic encephalopathy impairs fitness to drive. Hepatology. 2004;39:739-45.

38. Weissenborn K, Scholz, Hinrichs H, Wiltfang J, Schmidt FW, Kunkel H. Neuropsycological assessment of early hepatic encephalopathy. Eletroencephalogr Clin Neurophysiol 1990;75:289-95. 